\title{
Impact of gefitinib in early stage treatment on circulating cytokines and lymphocytes for patients with advanced non-small cell lung cancer
}

This article was published in the following Dove Press journal:

OncoTargets and Therapy

21 February 2017

Number of times this article has been viewed

\author{
Jin Sheng ${ }^{1-3, *}$ \\ Wenfeng Fang ${ }^{1-3, *}$ \\ Xia Liu $^{3}$ \\ Shan Xing ${ }^{1,2,4}$ \\ Jianhua Zhan ${ }^{1,2}$ \\ Yuxiang $\mathrm{Ma}^{1,2}$ \\ Yan Huang ${ }^{1-3}$ \\ Ningning Zhou ${ }^{1-3}$ \\ Hongyun Zhaol-3 \\ Li Zhang ${ }^{1-3}$
}

'State Key Laboratory of Oncology in South China, ${ }^{2}$ Collaborative Innovation Center for Cancer Medicine, ${ }^{3}$ Department of Medical Oncology, ${ }^{4}$ Department of Clinical Laboratory, Sun Yat-Sen University Cancer Center, Guangzhou, Guangdong, People's Republic of China

*These authors contributed equally to this work
Correspondence: Li Zhang

Department of Medical Oncology, Sun Yat-Sen University Cancer Center,

65I Dongfeng Road East, Guangzhou,

Guangdong 510060, People's

Republic of China

Tel +862087343458

Fax +86 2087343535

Email zhangli@sysucc.org.cn
Objectives: The impact of epidermal growth factor receptor (EGFR) tyrosine-kinase inhibitors (TKIs) on the human immune system remains undefined. This study illustrates the immunomodulatory effect of gefitinib in patients with advanced non-small cell lung cancer (NSCLC) and its relevant prognostic significance.

Patients and methods: Peripheral blood samples were collected from 54 patients at baseline and after 4 weeks of gefitinib treatment. Circulating lymphocyte populations and cytokine levels were measured. Pilot investigation of the impact of gefitinib on programmed cell death ligand-1 (PD-L1) expression was conducted by immunohistochemistry (IHC).

Results and conclusion: A significant increase of peripheral natural killer cells and interferongamma (INF- $\gamma$ ) after 4 weeks of gefitinib treatment ( $P=0.005$ and 0.02 , respectively). In addition, circulating interleukin (IL)-6 was significantly decreased, especially in patients sensitive to gefitinib $(P<0.001)$. Higher levels of IL-6 at baseline independently correlated with poorer progression-free survival. Experiments with NSCLC specimens illustrated that PD-L1 expression were downregulated after 4 weeks of gefitinib treatment. In summary, it was found that gefitinib treatment can alter circulating cytokines and lymphocytes. Dynamic changes of circulating lymphocytes, cytokines, and even PD-L1 IHC expression around gefitinib treatment support the specific immunomodulatory effect of this agent for advanced NSCLC.

Keywords: non-small cell lung cancer, gefitinib, PD-L1, lymphocyte, cytokine

\section{Introduction}

Recent insights into genetic aberrations and the role of the immune system in non-small cell lung cancer (NSCLC) have ushered in a new era of rapidly evolving targeted therapy and immune-based treatments. ${ }^{1-3}$ Tyrosine-kinase inhibitors (TKIs) targeting epidermal growth factor receptor (EGFR) are efficacious as targeted therapy for NSCLC. ${ }^{4-7}$ Although the progression-free survival (PFS) and the overall survival (OS) have been significantly improved, patients inevitably develop acquired resistance, and durable responses for advanced NSCLC have only been reported with immunologic therapy. 8,9

Very recent findings include the mechanism of immunoediting and the complexity of immune escape mechanisms in cancer. ${ }^{10}$ Programmed cell death protein-1 (PD-1) and its ligand-1 (PD-L1) are key immunological checkpoints mediating immune escape of cancer cells and limiting the anticancer immune response. ${ }^{11,12}$ Blocking PD-1 or PD-L1 can restore the functions of tumor-specific T cells, which will further be reactivated to initiate direct killing of tumor cells, and the secretion of immuno-stimulatory cytokines such as interferon-gamma (INF- $\gamma$ ), interleukin (IL)-2, and tumor necrosis 
factor alpha (TNF- $\alpha) \cdot{ }^{13,14}$ The immunological checkpoint PD-L1 is regarded as an important biomarker for tailoring immunotherapy. ${ }^{15}$

Increasing evidence has suggested that EGFR-TKIs may have important immunological functions. ${ }^{16}$ In fact, the enhanced antitumor effect seen in patients with skin rash may reflect a more functional antitumor immune response in these individuals. ${ }^{17}$ Surprisingly, the therapeutic efficacy of several targeted agents seems to rely in part on off-target mechanisms, some of which are mediated by the immune system. ${ }^{18}$ However, little is known about the immunoregulatory effects of EGFR-TKIs for NSCLC patients. This exploratory study illustrates the impact of gefitinib on peripheral inflammatory cytokines and lymphocytes. The potential prognostic value was also explored. Pilot investigation of PD-L1 expression was also performed on the basis of the available paired tissues pre- and posttreatment.

\section{Patients and methods}

\section{Patient characteristics and treatment scheme}

Patients diagnosed with NSCLC at the Cancer Center of Sun Yat-Sen University from March 2014 to March 2015 were screened for enrollment. The mutation status of EGFR was determined by amplification refractory mutation system-polymerase chain reaction. Patients were eligible to participate if they were between 18 and 75 years of age, pathologically diagnosed as advanced NSCLC, harbored EGFR-activating mutation, without T790M mutation, and treatment naive. Those patients who had 1) infection fever, inflammatory or autoimmune disease; 2) recent history of steroid, recent or current intake history of immunosuppressive drugs, opioid use or alcohol or illegal substance abuse; or 3) severe cardiac, respiratory, neurologic, or psychiatric diseases were excluded from this study.

Patients received gefitinib (IRESSA ${ }^{\circledR}$, AstraZeneca, Macclesfield, UK) treatment at the standard dose (orally, $250 \mathrm{mg}$ /day). Treatment interruptions and dose modifications were carried out according to the general recommendations. Computed tomography scans of the chest and abdomen and magnetic resonance imaging of the brain were performed at baseline, 4 weeks, and every 8 weeks thereafter or as clinically indicated for follow-up. Response evaluation was performed following the standard Response Evaluation Criteria in Solid tumors (RECIST version 1.0). Patients with a complete or partial response (PR) were regarded as objective response. PR refers to at least a $30 \%$ decrease in the sum of the longest diameter of target lesions. Systemic disease progression or the appearance of new lesions during treatment was considered disease progression.

Fasting blood samples were separately taken before (within 1 week) and 4 weeks after continuous gefitinib treatment. Serum samples were collected for cytokine analysis, and heparin plasma samples were collected for lymphocyte analysis.

\section{Cytokine measurements}

Blood samples were left to stand at room temperature for $30 \mathrm{~min}$. Serum was isolated from blood samples after centrifugation $(3,500 \mathrm{rpm}$ for $15 \mathrm{~min})$ and then stored at $-80^{\circ} \mathrm{C}$ for cytokine measurement. Cytokines in serum samples were measured with a BD CBA Th1/Th2 Cytokine Kit (Catalog No 551809; BD Biosciences, San Jose, CA, USA). The kit was used for the simultaneous detection of IL-2, IL-4, IL-6, INF- $\gamma$, and IL-10 in a single sample. This assay kit provided a mixture of capture beads with distinct fluorescence intensities that have been coated with capture antibodies specific for each cytokine. The assays were performed according to the manufacturer's instructions. Individual cytokine concentrations were determined by measuring the fluorescent intensities of the corresponding capture beads. Concentrations of all cytokines were reported as $\mathrm{pg} / \mathrm{mL}$.

\section{Lymphocyte phenotype analyses by flow cytometry}

CD3 FITC was used for the identification of T lymphocytes, CD16, and CD56 PE for the identification of natural killer (NK) lymphocytes, CD45 PerCP-Cy ${ }^{\mathrm{TM}} 5.5$ to allow for lymphocyte population gating, CD4 PE-Cy ${ }^{\mathrm{TM}} 7$ for detecting T-helper/inducer lymphocytes, CD19 APC to identify B lymphocytes, and CD8 APC-Cy7 for the identification of suppressor/cytotoxic T lymphocytes (BD Multitest ${ }^{\mathrm{TM}}$ 6-color TBNK reagent, Catalog No 644611; BD Biosciences). The cells were analyzed by a FACSCalibur flow cytometer using BD FACSDiva clinical software (BD Biosciences) as indicated by the manufacturer. The results corresponding to each lymphocyte were presented as percentages (\%) in human peripheral blood.

\section{Immunohistochemistry (IHC) analyses}

Paired, formalin-fixed, paraffin-embedded specimens were available from two patients with metastatic NSCLC. Both of them had a deletion of EGFR exon 19. The metastatic lesions were collected for comparison. The expression of PD-L1 was measured by immunohistochemical staining using a rabbit monoclonal antihuman antibody (E1L3NTM; Cell Signaling Technology, Danvers, MA, USA, 1:200). Two pathologists 
who were blinded to the clinical or pathological information of these patients independently assessed the expression of PD-L1. A semiquantitative H-score (maximum value of 300 corresponding to $100 \%$ of tumor cells positive for PD-L1 with an overall staining intensity score) was defined as the product of the percentage of stained cells and an intensity score ( 0 , absent; 1 , weak; 2 , moderate; and 3 , strong).

\section{Ethics statements}

The study protocol and patients' informed consent were approved by the Ethics Committee of Cancer Center of Sun Yat-Sen University (SYSUCC, Guangzhou, People's Republic of China). All the patients provided written informed consent to participate in this study. All procedures performed in the study involving human participants were in accordance with the ethical standards of the institutional and national research committee and with the 1964 Helsinki Declaration and its later amendments or comparable ethical standards. The studies conducted in laboratory were performed under exploratory research principles. The raw data can be provided per request.

\section{Statistical analysis}

Nonparametric quantitative data were presented as median and interquartile range (IQR). The Wilcoxon test was used for the comparison of paired nonparametric data. Pearson's chi-squared test or Fisher' exact test was used to assess the correlation between immunological parameters and the efficacy of gefitinib. PFS was calculated from the start of gefitinib treatment until disease progression, death, or the last follow-up. All of the statistical analyses were performed with SPSS 20.0 for Windows (IBM Corporation, Armonk, NY, USA). A two-sided $P$-value of $<0.05$ was considered statistically significant.

\section{Results}

The characteristics of 54 patients with advanced NSCLC receiving gefitinib are listed in Table 1 . Twenty-six patients (48\%) were male and 28 patients $(52 \%)$ were female. The median age was 57 (39-88) years old. The objective response rate (ORR) was $76 \%(\mathrm{n}=41$, all cases were partial remissions), and another nine patients (16\%) had stable disease. The median tumor size at baseline was $56.5 \mathrm{~mm}$ (IQR: $38.0-80.8 \mathrm{~mm}$ ). After gefitinib treatment, the median value decreased to 41.5 (IQR: 27.8-57.0). This change was significant according to Wilcoxon test $(P<0.001)$. The median PFS was 10.4 months (95\% confidence interval [CI], 10.1-10.7 months) with a median follow-up of 14.2 months. The percent of peripheral lymphocyte subsets at baseline
Table I The characteristics of enrolled NSCLC patients $(n=54)$

\begin{tabular}{ll}
\hline Parameter case & N (\%) \\
\hline Gender & \\
Female & $28(52)$ \\
Male & $26(48)$ \\
Smoking & \\
$\quad$ Never & $36(67)$ \\
Ever & $18(33)$ \\
Stage & \\
IIIB & $3(6)$ \\
IV & $51(94)$ \\
Pathology & \\
ADC & $48(89)$ \\
Non-ADC & $6(11)$ \\
EGFR mutation status & \\
Exon I9 del & $33(61)$ \\
Exon 2I L858R & $17(32)$ \\
Others* & $4(7)$ \\
Gefitinib efficacy & \\
Partial response & $41(76)$ \\
Stable disease & $9(17)$ \\
Progression disease & $4(7)$ \\
\hline
\end{tabular}

Notes: Non-ADC includes four squamous carcinoma and two adenosquamous carcinoma. *Other EGFR-activating mutations without EGFR exon T790M. Two cases harbor exon 18 (Gly719Ser and Gly719Ala) and another two cases with exon 21 (Leu86IGIn), respectively.

Abbreviations: ADC, adenocarcinoma; EGFR, epidermal growth factor receptor; NSCLC, non-small cell lung cancer.

and after 4 weeks of gefitinib treatment was determined by flow cytometry. The dynamic changes are presented in Figure 1. The percent of total $\mathrm{T}$ cells $\left(\mathrm{CD} 4^{+} \mathrm{T}\right.$ cells) was significantly decreased after 4 weeks $(P=0.003)$. An inverse relationship was found in effector $\mathrm{T}$ cells $\left(\mathrm{CD} 8^{+} \mathrm{T}\right.$ cells $)$; however, the difference was not significant $(P=0.48)$. Additionally, a pronounced induction of peripheral NK cells $\left(\mathrm{CD} 3^{-} \mathrm{CD} 16^{+} \mathrm{CD} 56^{+}\right)$was detected after gefitinib treatment $(P=0.005)$, which was considered as an augmentation of innate immunity. Whether regulatory $\mathrm{CD} 4{ }^{+} \mathrm{CD} 25^{+} \mathrm{T}$ cells were influenced by gefitinib treatment was also explored. A slight increase in total $\mathrm{T}$ cells was induced by gefitinib, but the change was not statistically significant $(P=0.18)$.

Whether gefitinib could modulate the peripheral levels of pro-inflammatory cytokines was further explored. The levels of circulating IL-6 were significantly decreased after gefitinib treatment $(P<0.001$, Figure 2A). Moreover, 4 weeks later, a significant increase of peripheral INF- $\gamma$ levels was detected $(P=0.02$, Figure 2D). In order to elucidate the influence of the treatment on the dynamic changes of INF- $\gamma$ and IL- 6 levels, the results were distinguished according to gefitinib efficacy. Significant changes in IL-6 (Figure 2B and C) and INF- $\gamma$ (Figure 2E and F) were present only in the response group. The correlation between the alteration in immunological parameters and the efficacy of gefitinib was further analyzed. 
A

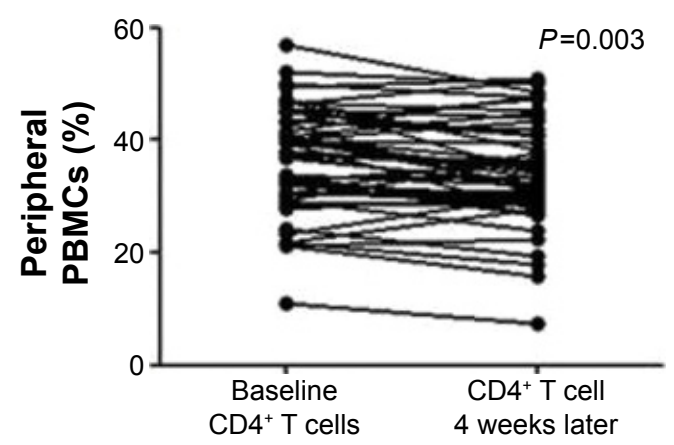

C

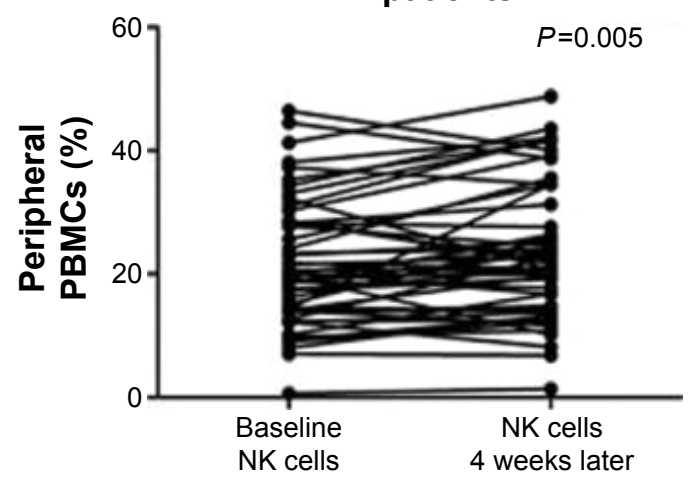

B

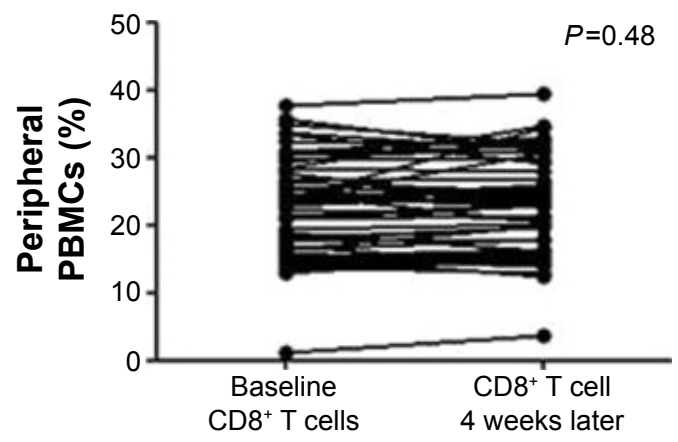

D

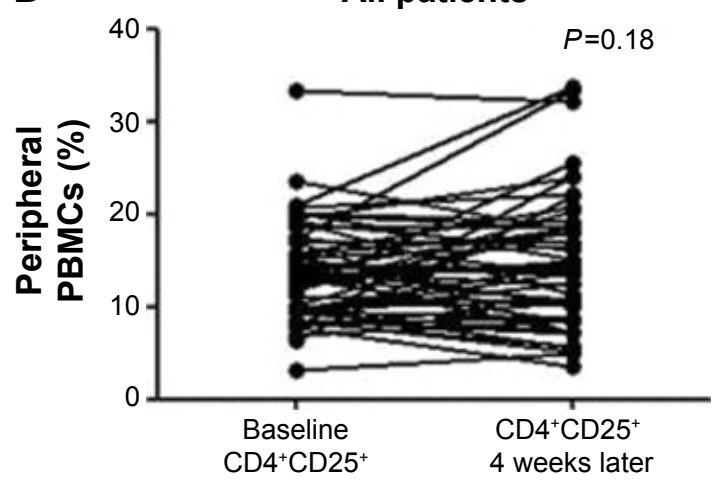

Figure I Gefitinib-induced changes of the peripheral lymphocyte subsets: (A) CD4 ${ }^{+} \mathrm{T}$ cells, (B) CD8 ${ }^{+} \mathrm{T}$ cells, (C) NK cells, and (D) CD4 CD25+ T cells. Note: The changes of lymphocyte in terms of percent of PBMCs were analyzed for all patients $(n=54)$ at baseline and 4 weeks after gefitinib treatment. Abbreviation: PBMC, peripheral blood mononuclear cell.
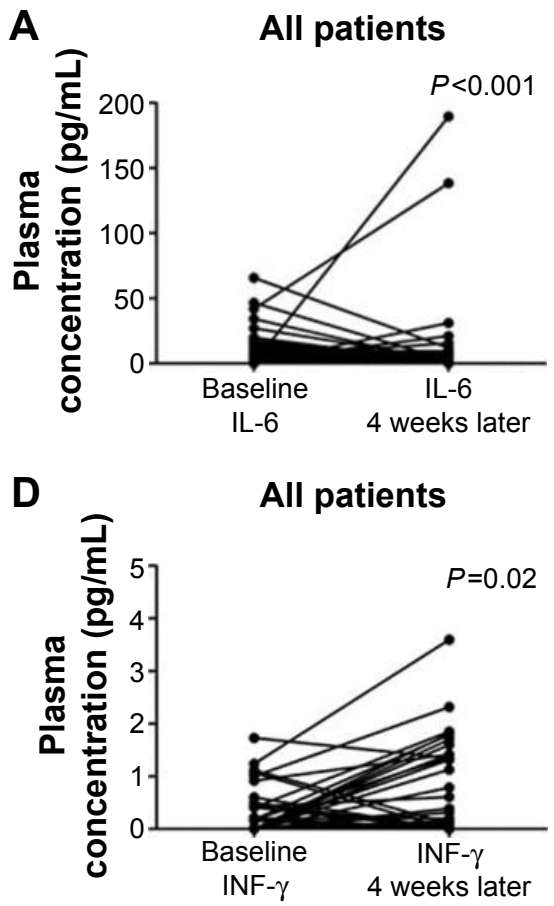

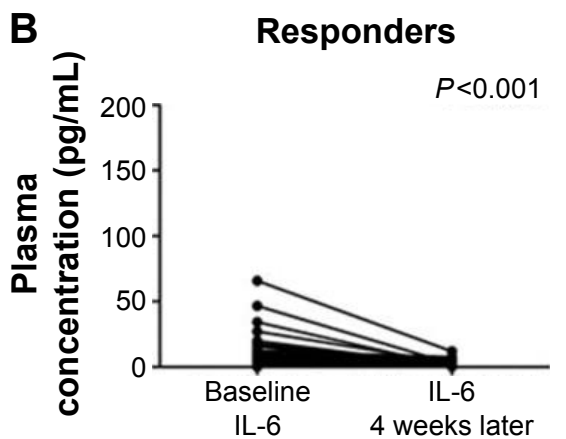

E

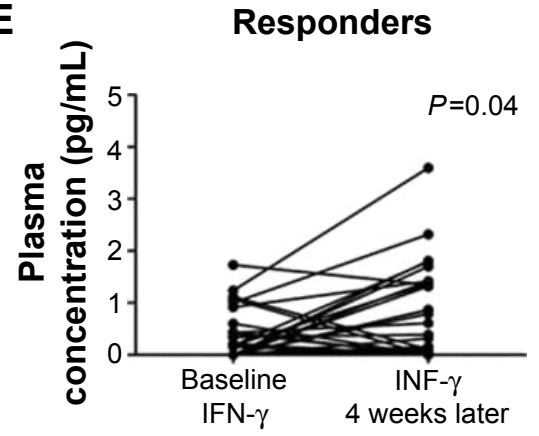

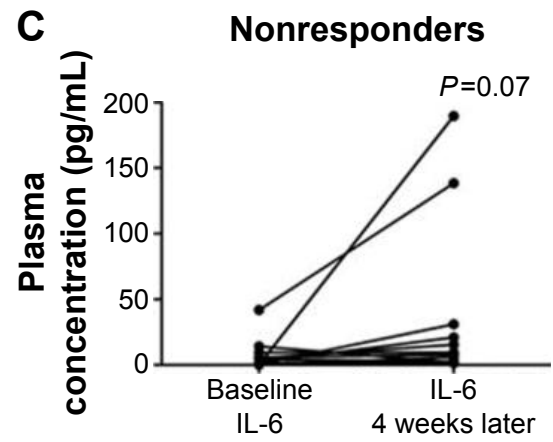

$\mathbf{F}$

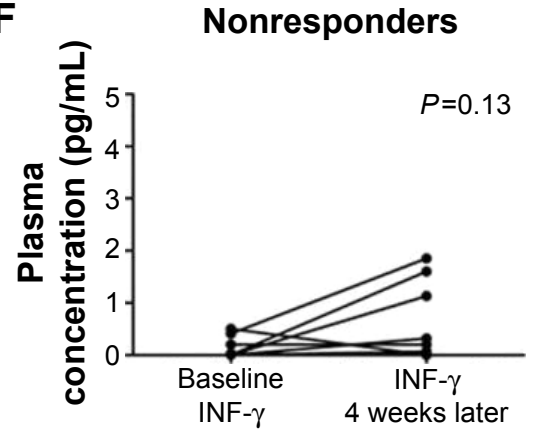

Figure 2 Gefitinib-induced decrease of circulating IL-6 and increase of INF- $\gamma$.

Notes: (A) IL-6, all patients. (B) IL-6, responders to gefitinib. (C) IL-6, nonresponders to gefitinib. (D) INF- $\gamma$, all patients. (E) INF- $\gamma$, responders to gefitinib. (F) nonresponders to gefitinib. All patients $(n=54)$; responders to gefitinib $(n=4 I)$; nonresponders to gefitinib $(n=\mid 3)$.

Abbreviations: IL, interleukin; INF- $\gamma$, interferon- $\gamma$. 
The dynamic feature was classified according to the change caused by the treatment. No apparent changes in circulating IL-2, IL-4, and IL-10 were detected after gefitinib treatment ( $P$-values were $0.12,0.17$, and 0.70 , respectively; Figure $\mathrm{S} 1$ ). Despite no significant association between changes in IL-2, IL-4, IL-10, and INF- $\gamma$ and treatment efficacy, reduced IL-6 levels were significantly correlated with objective response to gefitinib (Table 2).

The prognostic significance of circulating IL-6 on PFS was then investigated. Changes in plasma IL-6 during gefitinib treatment were classified into reduced or nonreduced groups. Although reduced IL-6 levels might predict a response to gefitinib, it failed to present as an independent predictor for PFS by Cox proportional hazards regression models (Table S1). In order to demonstrate this finding, baseline and posttreatment plasma levels were also analyzed. In each circumstance, median values were used as the cutoff between the higher and lower groups separately. KaplanMeier survival curves are presented in Figure 3. Higher

Table 2 The dynamic change of several immunological parameters and the efficacy of gefitinib

\begin{tabular}{|c|c|c|c|c|}
\hline Parameters & $\begin{array}{l}\text { Response } \\
(\mathrm{N})\end{array}$ & $\begin{array}{l}\text { Nonresponse } \\
(\mathrm{N})\end{array}$ & $\chi^{2}$ & $P$-value \\
\hline \multicolumn{5}{|l|}{ IL-2 } \\
\hline Reduced & 15 & 5 & 0.02 & 1.00 \\
\hline Nonreduced & 26 & 8 & & \\
\hline \multicolumn{5}{|l|}{$\mathrm{IL}-4$} \\
\hline Reduced & 13 & 6 & 0.90 & 0.51 \\
\hline Nonreduced & 28 & 7 & & \\
\hline \multicolumn{5}{|l|}{ IL-6 } \\
\hline Reduced & 31 & 5 & 6.13 & 0.02 \\
\hline Nonreduced & 10 & 8 & & \\
\hline \multicolumn{5}{|l|}{ IL-I0 } \\
\hline Elevated & 18 & 4 & - & $0.52^{\mathrm{a}}$ \\
\hline Nonelevated & 23 & 9 & & \\
\hline \multicolumn{5}{|l|}{ INF- $\gamma$} \\
\hline Elevated & 19 & 3 & - & $0.20^{\mathrm{a}}$ \\
\hline Nonelevated & 22 & 10 & & \\
\hline \multicolumn{5}{|c|}{$\mathrm{CD} 4^{+} \mathrm{CD} 25^{+} \mathrm{T}$ cells } \\
\hline Reduced & 23 & 5 & 1.23 & 0.27 \\
\hline Nonreduced & 18 & 8 & & \\
\hline \multicolumn{5}{|c|}{ Natural killer cells } \\
\hline Elevated & 24 & 11 & 2.94 & 0.09 \\
\hline Nonelevated & 17 & 2 & & \\
\hline \multicolumn{5}{|l|}{$\mathrm{CD}^{+} \mathrm{T}$ cells } \\
\hline Reduced & 28 & 10 & - & $0.73^{a}$ \\
\hline Nonreduced & 13 & 3 & & \\
\hline \multicolumn{5}{|l|}{$\mathrm{CD}^{+} \mathrm{T}$ cells } \\
\hline Elevated & 22 & 7 & $<0.001$ & 1.00 \\
\hline Nonelevated & 19 & 6 & & \\
\hline
\end{tabular}

Notes: The dynamic feature was classified according to the change trend that was observed in the study. ${ }^{2} P$-value was calculated with Fisher's exact test; all others were calaculated with Pearson chi-squared test.

Abbreviations: IL, interleukin; INF- $\gamma$, interferon- $\gamma$.

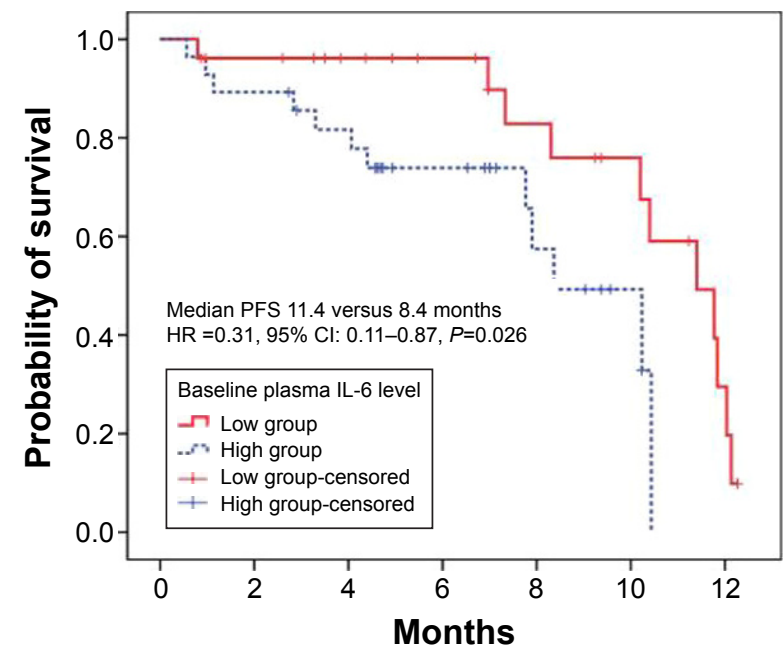

Figure 3 The prognostic value of IL-6 for PFS.

Note: Higher level $(\geq 5.5 \mathrm{pg} / \mathrm{mL})$ versus lower level $(<5.5 \mathrm{pg} / \mathrm{mL})$ of IL- 6 at baseline. Abbreviations: $\mathrm{Cl}$, confidence interval; HR, hazard ratio; IL, interleukin; PFS, progression-free survival.

levels of IL-6 at baseline correlated significantly with shorter PFS compared to the group with lower levels of IL-6 at baseline, with a median PFS of 8.4 and 11.4 months, respectively (Table 3). However, this difference was not as large in posttreatment plasma IL-6 levels, in which the median PFS was 10.2 and 11.4 months, respectively (Table S2 and Figure S2).

Finally, a pilot investigation was conducted to verify whether the expression of PD-L1, an immunomodulatory checkpoint molecule, could be altered by gefitinib in the early treatment stage. The results revealed that gefitinib treatment reduced the expression of PD-L1 4 weeks later compared to that at baseline. The H-score of PD-L1 changed from 120 and 80 to 40 in both the cases. Images of gefitinib-treated tumor cells stained for PD-L1 are presented in Figure S3.

\section{Discussion}

In the current era of rapidly evolving targeted therapies and immune-based treatments, limited evidence for gefitinib or erlotinib as immunomodulatory drugs remains. Although some insight into the interaction between EGFR-TKIs and immunological checkpoints has been reported, little is known about the immunoregulatory effects of these targeted agents in vivo. This study demonstrated for the first time that gefitinib could alter the composition of peripheral lymphocytes and the levels of circulating pro-inflammatory cytokines. The increased innate immunity, increased systemic INF- $\gamma$, and reduced circulating IL- 6 suggested that an immune-related mechanism may be involved in mediating the anticancer effects of gefitinib. In addition, the immunomodulatory 
Table 3 Univariate and multivariate survival analyses of 54 NSCLC patients

\begin{tabular}{|c|c|c|c|c|c|c|c|c|}
\hline \multirow[t]{3}{*}{ Variables } & \multirow[t]{3}{*}{ Category } & \multirow{3}{*}{$\begin{array}{l}\text { Median PFS } \\
\text { (months) }\end{array}$} & \multicolumn{6}{|c|}{ PFS analyses } \\
\hline & & & \multicolumn{3}{|c|}{ Univariate analyses } & \multicolumn{3}{|c|}{ Multivariate analyses } \\
\hline & & & HR & $95 \% \mathrm{Cl}$ & $P$-value & HR & $95 \% \mathrm{Cl}$ & $P$-value \\
\hline Gender & Female/male & II.8 vs 7.9 & 0.25 & $0.09-0.69$ & 0.008 & 0.24 & $0.07-0.83$ & 0.025 \\
\hline Age & $<57 / \geq 57$ years & II .4 vs 7.9 & 0.39 & $0.17-0.90$ & 0.028 & 0.24 & $0.08-0.66$ & 0.006 \\
\hline Smoking history & No/yes & I0.4 vs 7.9 & 0.35 & $0.14-0.91$ & 0.031 & 1.58 & $0.50-5.26$ & 0.452 \\
\hline EGFR status & Exon $19 \mathrm{del} /$ others & II.8 vs 8.4 & 0.31 & $0.12-0.79$ & 0.014 & 0.34 & $0.13-0.91$ & 0.032 \\
\hline IL-6 level* & Lower/higher & II.4 vs 8.4 & 0.31 & $0.11-0.87$ & 0.026 & 0.19 & $0.05-0.66$ & 0.009 \\
\hline Response to gefitinib & $\mathrm{PR} /$ not $\mathrm{PR}$ & 10.4 vs 10.2 & 0.45 & $0.18-1.12$ & 0.086 & 0.17 & $0.05-0.53$ & 0.002 \\
\hline
\end{tabular}

Note: *Patients were dichotomized on the basis of the median value $(5.5 \mathrm{pg} / \mathrm{mL})$ of baseline plasma IL-6.

Abbreviations: $\mathrm{Cl}$, confidence interval; EGFR, epidermal growth factor receptor; HR, hazard ratio; IL, interleukin; NSCLC, non-small cell lung cancer; PFS, progression-free survival; PR, partial response.

effects were also reflected in the downregulation of PD-L1 expression after 4 weeks of gefitinib treatment.

NK cells are key components of innate immunity against viral infection and neoplastic cells. ${ }^{19} \mathrm{NK}$ cells can eliminate cancer cells via direct killing, induction of apoptosis, or INF- $\gamma$ secretion. ${ }^{20,21} \mathrm{He}$ et $\mathrm{al}^{22}$ reported that gefitinib could greatly enhance NK-cell cytotoxicity by restoring receptor-ligand interactions between NK cells and human lung cancer cells with EGFR L858R and T790M resistance mutations. Moreover, NK-cell-based immunotherapy is regarded as a promising way to eliminate tumor cells. ${ }^{23}$ The results of this study were in accordance with the previous reports. After 4 weeks of gefitinib treatment, the percentage of NK cells was significantly increased from $21.3 \%$ to $23.7 \%$ ( $P=0.005$; Table S3). This change in the innate immune system was likely due to enhanced receptor-ligand interactions described earlier.

Moreover, the impact of gefitinib on adoptive cellular immunoreaction was also explored. Peripheral percentages of $\mathrm{CD}^{+} \mathrm{T}$ cells were significantly decreased after 4 weeks of gefitinib treatment, whereas no apparent alteration in the percentage of effector $\mathrm{T}$ cells was observed. A research study previously revealed that erlotinib could impair T-cell-mediated immune responses both in vitro and in vivo by inhibiting T-cell proliferation and activation. ${ }^{24}$ This immunosuppressive activity was believed to be due to the downregulation of the c-Raf/ERK cascade and the Akt signaling pathway. The Ras/Raf/ERK and PI3K/Akt pathways are critical in the signaling networks that regulate cell proliferation, differentiation, and survival. ${ }^{25,26}$ Of note, drug sensitivity to gefitinib is closely correlated with EGFRdependent ERK1/2 and Akt activation. ${ }^{27}$ Our controversial finding was presumably due to different degrees of inhibition mediated by gefitinib or different mechanisms than the one by which erlotinib acts against the T-cell-mediated immune response. Despite the complexity of the human immune system, evidence at clinical level to demonstrate the immunomodulatory impact of gefitinib has been provided.

Immune cells have a broad impact on tumor initiation, progression, and drug resistance. Several proinflammatory cytokines are considered to be vital in the aforementioned process. ${ }^{28,29}$ INF- $\gamma$ and IL-6, for instance, are pleiotropic cytokines with complex functions in various immune and inflammatory reactions..$^{30,31}$ Moreover, INF- $\gamma$ is an important effector cytokine produced by NK cells, and it is vital for the immune surveillance of tumors. ${ }^{32}$ IL-6 has been reported to be associated with tumor progression, and it acts to inhibit cancer cell apoptosis and stimulate angiogenesis. ${ }^{33}$ IL-6 was shown to induce an epithelial-to-mesenchymal transition (EMT) and promote metastasis in various human cancers. ${ }^{34-36}$ Several studies have reported resistance to anti-NSCLC agents that is mediated by IL- $6 .{ }^{36,37}$ TGF- $\beta /$ IL- 6 axis mediates selective and adaptive mechanisms of resistance to erlotinib in lung cancer. ${ }^{37}$ In addition, previous data have already suggested a statistically significant difference between lung cancer patients and the healthy control group regarding circulating IL- $6 .{ }^{38}$ Results from Songur et al showed a relationship between elevated serum IL-6 levels and malnutrition and bad prognosis in patients with advanced NSCLC. ${ }^{39}$ The results of the present study also demonstrated that gefitinib significantly increased systemic levels of INF- $\gamma$ and decreased the circulating levels of IL-6. This alteration was more apparent in those that were sensitive to gefitinib. There is evidence for circulating IL-6 as a survival predictor in advanced NSCLC patients treated with chemotherapy. ${ }^{40} \mathrm{~A}$ similar result was found in terms of PFS for NSCLC patients receiving gefitinib. However, the differences found in this study were only at higher baseline levels of IL-6, which correlated with shorter PFS, whereas the change types (reduced versus nonreduced) and posttreatment levels were not independent predictors. However, this study cannot conclude whether the changes 
are specific to gefitinib or a universal phenomenon when receiving chemotherapeutic agents.

Indeed, preclinical data have demonstrated that the continuing activation of EGFR contributes to the upregulation of $p$-STAT3, which leads to the increased expression of IL- $6 .{ }^{41}$ The blocking of EGFR activation induced by TKI consequently generates the downregulation of IL-6. Given the biologic rationale for therapeutic anti-IL-6 activity and preliminary clinical evidence that targeted anti-IL-6 antibodies are well tolerated in cancer patients, the findings of this study provide further insight into a potential strategy for improving gefitinib anticancer efficacy and prolonging PFS by combining an anti-IL- 6 agent with gefitinib. ${ }^{42,43}$

Along with the enthusiastic promotion of PD-1/PD-L1 checkpoint blockades, most biomarker investigation has focused on the tumor microenvironment, especially the immunohistochemical expression of PD-L1. ${ }^{44}$ This study verified that EGFR-TKIs, such as gefitinib, can downregulate tumor PD-L1 expression in patients with NSCLC. This validation was concordant with previously published data. ${ }^{13-15}$ Erlotinib could downregulate PD-L1 expression in cell lines with activating EGFR mutations but not in those with wild type PD-L1. ${ }^{13}$ Meanwhile, our previous work showed that inhibiting EGFR with EGFR-TKIs could attenuate the inhibition of $\mathrm{T}$ cells mediated by PD-L1 and enhance the production of INF- $\gamma .{ }^{14}$ Besides, the changes of aforementioned circulating parameters were probably a consequence of the influence of mediators released from disintegrated cancer cells or enhanced immune system during gefitinib treatment. Therefore, the immunomodulatory effect may be due to the alteration of the PD-1/PD-L1 interaction caused by downregulating PD-L1 expression and increasing INF- $\gamma$ levels and NK-cell activation. ${ }^{14,45}$ In addition, the results of the present study demonstrated that PD-L1 expression is not constant during EGFR-TKIs treatment. The clinical significance here is that physicians should be cautious while applying PD-L1 status or expression level in pretreatment specimens as a prognostic factor or biomarker for immunotherapy. At present, targeted therapy directed at oncogenic signaling pathways is an attractive candidate in combination with immune checkpoint blockade agents. ${ }^{42,46}$ Nevertheless, synergistic antitumor effect was not observed with combined EGFR-TKIs and an anti-PD-1 antibody treatment in a coculture system. ${ }^{14}$ The positive immunoregulatory effect of gefitinib observed in the present study weakens the demand for the combination of gefitinib with immunotherapy. However, more investigation and prospective clinical studies are needed to verify this hypothesis.
This study was limited by its exploratory nature and the relatively small number of participants. Paired tissues for PD-L1 detection were a limited resource, which restricts these experiments as a pilot investigation. In addition, the causality between PD-L1 reduction and the alteration of lymphocytes or cytokines is not identified in the present work. Despite these limitations, a paired comparison was used during early gefitinib treatment and, for the first time, the specific immunomodulatory effects of gefitinib were detected.

Collectively, this study demonstrated that gefitinib treatment at early stages leads to distinct changes of circulating lymphocytes, cytokines, and even PD-L1 IHC expression around treatment. All these data support that certain immunomodulatory effects exist for gefitinib in advanced NSCLC.

\section{Acknowledgments}

We thank the patients who participated in this study and their families; the medical, nursing, and research staffs at the study centers. Special thanks to Dr Yunpeng Yang, Yaxiong Zhang, and Yuanyuan Zhao from Medical Oncology, Cancer Center of Sun Yat-Sen University and Dr Nan Chen from Fifth Affiliated Hospital of Sun Yat-Sen University, for their good suggestion and help for improving the design and conduction. This study was supported by National Natural Science Foundation of China (Grant No 81372502, 81572659), Guangzhou Science and Technology Project (Grant No 201400000001-2), the Young Teacher Training Program of Sun Yat-Sen University (Grant No 14ykpy38), Physician-Scientist Cultivation Project of Sun Yat-Sen University Cancer Center (Grant No 04140701), Wu Jieping Medical Foundation Project (Grant No 320.6750.131), and Cancer Research Foundation of CSCO-Merck Serono (Y-MT2015-038). All the funders had no role in study design, data collection and analysis, decision to publish, or preparation of the manuscript.

\section{Author contributions}

LZ, WFF, and JS designed the study. XL collected the blood samples, and SX and JHZ conducted the experiment. YXM collected the raw data and with NNZ together analyzed it. JS, WFF, LZ, YH, and HYZ wrote the manuscript. All authors contributed toward data analysis, drafting and critically revising the paper and agree to be accountable for all aspects of the work.

\section{Disclosure}

The authors report no conflicts of interest in this work. 


\section{References}

1. Yang JC, Wu YL, Schuler M, et al. Afatinib versus cisplatin-based chemotherapy for EGFR mutation-positive lung adenocarcinoma (LUXLung 3 and LUX-Lung 6): analysis of overall survival data from two randomised, phase 3 trials. Lancet Oncol. 2015;16:141-151.

2. de Visser KE, Eichten A, Coussens LM. Paradoxical roles of the immune system during cancer development. Nat Rev Cancer. 2006;6:24-37.

3. Topalian SL, Hodi FS, Brahmer JR, et al. Safety and activity of antiPD-L1 antibody in patients with advanced cancer. $N$ Engl $J$ Med. 2012;366:2455-2465.

4. Zhou C, Wu YL, Chen G, et al. Erlotinib versus chemotherapy as first-line treatment for patients with advanced EGFR mutation-positive non-smallcell lung cancer (OPTIMAL, CTONG-0802): a multicentre, open-label, randomised, phase 3 study. Lancet Oncol. 2011;12:|735-742.

5. Mok TS, Wu YL, Thongprasert S, et al. Gefitinib or carboplatin-paclitaxel in pulmonary adenocarcinoma. N Engl J Med. 2009;361:947-957.

6. Shepherd FA, Rodrigues Pereira J, Ciuleanu T, et al. Erlotinib in previously treated non-small-cell lung cancer. $N$ Engl J Med. 2005; 353:123-132.

7. Sequist LV, Martins RG, Spigel D, et al. First-line gefitinib in patients with advanced non-small-cell lung cancer harboring somatic EGFR mutations. J Clin Oncol. 2008;26:2442-2449.

8. Zielinski C, Knapp S, Mascaux C, Hirsch F. Rationale for targeting the immune system through checkpoint molecule blockade in the treatment of non-small-cell lung cancer. Ann Oncol. 2013;24:1170-1179.

9. Forde PM, Reiss KA, Zeidan AM, Brahmer JR. What lies within: novel strategies in immunotherapy for non-small cell lung cancer. Oncologist. 2013;18:1203-1213.

10. Aerts JG, Lievense LA, Hoogsteden HC, Hegmans JP. Immunotherapy prospects in the treatment of lung cancer and mesothelioma. Transl Lung Cancer Res. 2014;3:34-45.

11. Korman AJ, Peggs KS, Allison JP. Checkpoint blockade in cancer immunotherapy. Adv Immunol. 2006;90:297-339.

12. McDermott DF, Atkins MB. PD-1 as a potential target in cancer therapy. Cancer Med. 2013;2:662-673.

13. Azuma K, Ota K, Kawahara A, et al. Association of PD-L1 overexpression with activating EGFR mutations in surgically resected nonsmallcell lung cancer. Ann Oncol. 2014;25:1935-1940.

14. Chen N, Fang W, Zhan J, et al. Upregulation of PD-L1 by EGFR activation mediates the immune escape in EGFR-driven NSCLC: implication for optional immune targeted therapy for NSCLC patients with EGFR mutation. J Thorac Oncol. 2015;10:910-923.

15. Taube JM, Klein A, Brahmer JR, et al. Association of PD-1, PD-1 ligands, and other features of the tumor immune microenvironment with response to anti-PD-1 therapy. Clin Cancer Res. 2014;20:5064-5074.

16. Brooks MB. Erlotinib and gefitinib, epidermal growth factor receptor kinase inhibitors, may treat non-cancer-related tumor necrosis factoralpha mediated inflammatory diseases. Oncologist. 2013;18:e3-e5.

17. Mohamed MK, Ramalingam S, Lin Y, Gooding W, Belani CP. Skin rash and good performance status predict improved survival with gefitinib in patients with advanced non-small cell lung cancer. Ann Oncol. 2005;16:780-785.

18. Galluzzi L, Senovilla L, Zitvogel L, Kroemer G. The secret ally: immunostimulation by anticancer drugs. Nat Rev Drug Discov. 2012;11:215-233.

19. Lodoen M, Ogasawara K, Hamerman JA, et al. NKG2D-mediated natural killer cell protection against cytomegalovirus is impaired by viral gp40 modulation of retinoic acid early inducible 1 gene molecules. J Exp Med. 2003;197:1245-1253.

20. Miller JS. The biology of natural killer cells in cancer, infection, and pregnancy. Exp Hematol. 2001;29:1157-1168.

21. Raulet DH, Guerra N. Oncogenic stress sensed by the immune system: role of natural killer cell receptors. Nat Rev Immunol. 2009;9:568-580.

22. He S, Yin T, Li D, et al. Enhanced interaction between natural killer cells and lung cancer cells: involvement in gefitinib-mediated immunoregulation. $J$ Transl Med. 2013;11:186.

23. Leemhuis T, Wells S, Scheffold C, Edinger M, Negrin RS. A phase I trial of autologous cytokine-induced killer cells for the treatment of relapsed Hodgkin disease and non-Hodgkin lymphoma. Biol Blood Marrow Transplant. 2005;11:181-187.
24. Luo Q, Gu Y, Zheng W, et al. Erlotinib inhibits T-cell-mediated immune response via down-regulation of the c-Raf/ERK cascade and Akt signaling pathway. Toxicol Appl Pharmacol. 2011;251:130-136.

25. Gollob JA, Wilhelm S, Carter C, Kelley SL. Role of Raf kinase in cancer: therapeutic potential of targeting the Raf/MEK/ERK signal transduction pathway. Semin Oncol. 2006;33:392-406.

26. Fresno Vara JA, Casado E, de Castro J, Cejas P, Belda-Iniesta C, González-Barón M. PI3K/Akt signalling pathway and cancer. Cancer Treat Rev. 2004;30:193-204.

27. Ono M, Kuwano M. Molecular mechanisms of epidermal growth factor receptor (EGFR) activation and response to gefitinib and other EGFRtargeting drugs. Clin Cancer Res. 2006;12:7242-7251.

28. O'Callaghan DS, O'Donnell D, O'Connell F, O'Byrne KJ. The role of inflammation in the pathogenesis of non-small cell lung cancer. $J$ Thorac Oncol. 2010;5:2024-2036.

29. Grivennikov SI, Karin M. Inflammatory cytokines in cancer: tumour necrosis factor and interleukin 6 take the stage. Ann Rheum Dis. 2011; 70(Suppl 1):i104-i108.

30. Boehm U, Klamp T, Groot M, Howard JC. Cellular responses to interferon-gamma. Аппи Rev Immunol. 1997;15:749-795.

31. Hodge DR, Hurt EM, Farrar WL. The role of IL-6 and STAT3 in inflammation and cancer. Eur J Cancer. 2005;41:2502-2012.

32. Kaplan DH, Shankaran V, Dighe AS, et al. Demonstration of an interferon gamma-dependent tumor surveillance system in immunocompetent mice. Proc Natl Acad Sci U S A. 1998;95:7556-7561.

33. Lukaszewicz M, Mroczko B, Szmitkowski M. Clinical significance of interleukin-6 (IL-6) as a prognostic factor of cancer disease. Pol Arch Med Wewn. 2007;117:247-251.

34. Sullivan NJ, Sasser AK, Axel AE, et al. Interleukin-6 induces an epithelial-mesenchymal transition phenotype in human breast cancer cells. Oncogene. 2009;28:2940-2947.

35. Colomiere M, Ward AC, Riley C, et al. Cross talk of signals between EGFR and IL-6R through JAK2/STAT3 mediate epithelialmesenchymal transition in ovarian carcinomas. Br J Cancer. 2009; 100:134-144.

36. Thomson S, Buck E, Petti F, et al. Epithelial to mesenchymal transition is a determinant of sensitivity of non-small-cell lung carcinoma cell lines and xenografts to epidermal growth factor receptor inhibition. Cancer Res. 2005;65:9455-9462.

37. Yao Z, Fenoglio S, Gao DC, et al. TGF-beta IL-6 axis mediates selective and adaptive mechanisms of resistance to molecular targeted therapy in lung cancer. Proc Natl Acad Sci U S A. 2010;107: $15535-15540$

38. Song XY, Zhou SJ, Xiao N, et al. Research on the relationship between serum levels of inflammatory cytokines and non-small cell lung cancer. Asian Pac J Cancer Prev. 2013;14:4765-4768.

39. Songur N, Kuru B, Kalkan F, Ozdilekcan C, Cakmak H, Hizel N. Serum interleukin-6 levels correlate with malnutrition and survival in patients with advanced non-small cell lung cancer. Tumori. 2004; 90:196-200.

40. Chang $\mathrm{CH}$, Hsiao $\mathrm{CF}$, Yeh YM, et al. Circulating interleukin-6 level is a prognostic marker for survival in advanced nonsmall cell lung cancer patients treated with chemotherapy. Int J Cancer. 2013;132: 1977-1985.

41. Harada D, Takigawa N, Kiura K. The role of STAT3 in non-small cell lung cancer. Cancers (Basel). 2014;6:708-722.

42. Guo Y, Xu F, Lu T, Duan Z, Zhang Z. Interleukin-6 signaling pathway in targeted therapy for cancer. Cancer Treat Rev. 2012;38:904-910.

43. Trikha M, Corringham R, Klein B, Rossi JF. Targeted anti-interleukin-6 monoclonal antibody therapy for cancer: a review of the rationale and clinical evidence. Clin Cancer Res. 2003;9:4653-4665.

44. Postow MA, Callahan MK, Wolchok JD. Immune checkpoint blockade in cancer therapy. J Clin Oncol. 2015;33:1974-1982.

45. Cerwenka A, Falk CS, Watzl C. NK cells from basic research to cancer therapy. Eur J Immunol. 2007;37:1161-1164.

46. Vanneman M, Dranoff G. Combining immunotherapy and targeted therapies in cancer treatment. Nat Rev Cancer. 2012;12:237-251. 


\section{Supplementary materials}
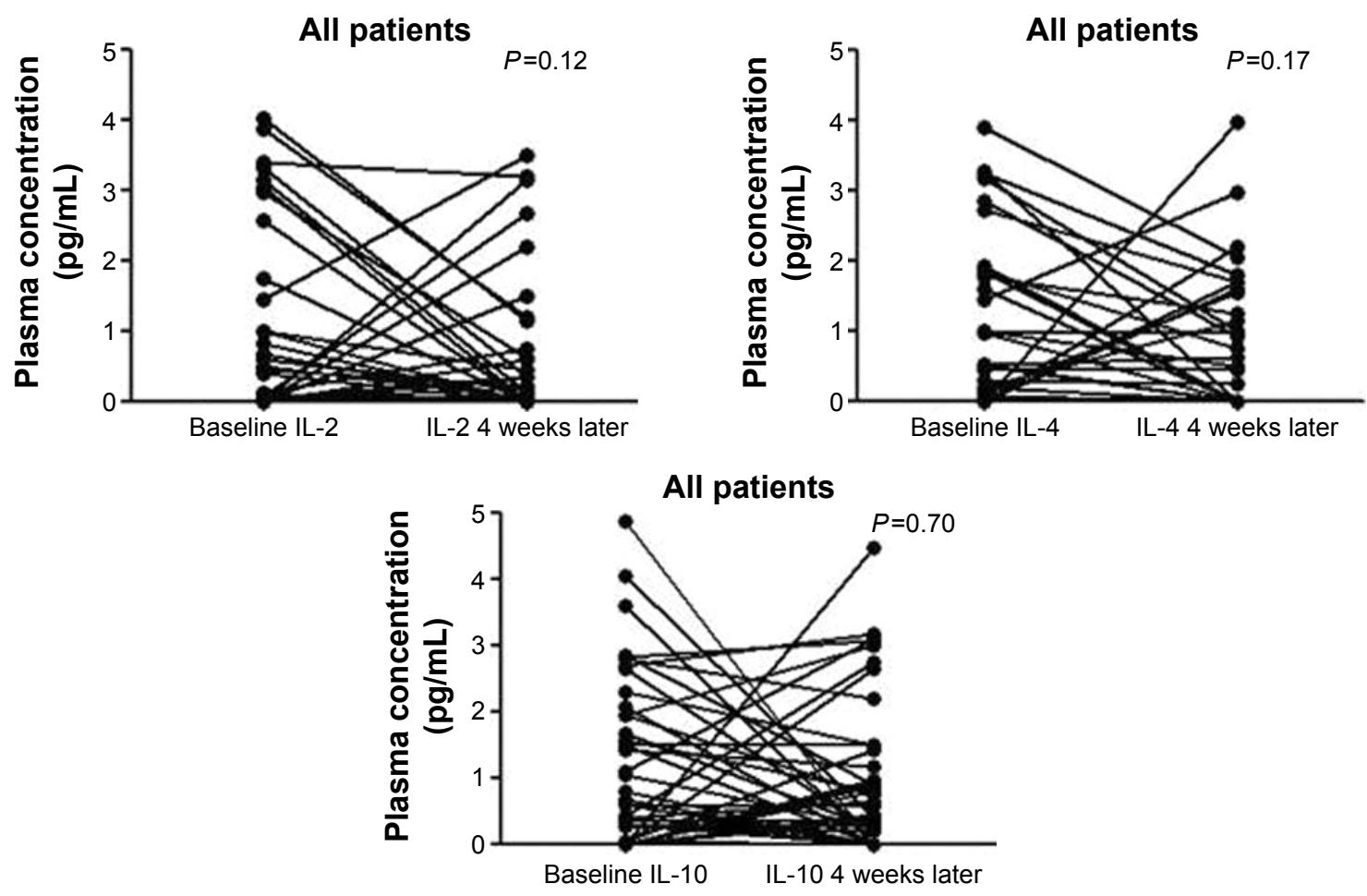

Figure SI The changes of several circulating cytokines induced by gefitinib treatment.

Note: These were analyzed for all patients $(n=54)$ with a baseline and 4 weeks after gefitinib treatment.

Abbreviation: IL, interleukin.

Table SI Univariate and multivariate survival analyses involving plasma IL-6 change types

\begin{tabular}{|c|c|c|c|c|c|c|c|}
\hline \multirow[t]{3}{*}{ Variables } & \multirow[t]{3}{*}{ Category } & \multicolumn{6}{|c|}{ Progression-free survival analyses } \\
\hline & & \multicolumn{3}{|c|}{ Univariate analysis } & \multicolumn{3}{|c|}{ Multivariate analysis } \\
\hline & & HR & $95 \% \mathrm{Cl}$ & $P$-value & HR & $95 \% \mathrm{Cl}$ & $P$-value \\
\hline Gender & Female/male & 0.25 & $0.09-0.69$ & 0.008 & 0.30 & $0.09-1.02$ & 0.055 \\
\hline Age & $<57 / \geq 57$ years & 0.39 & $0.17-0.90$ & 0.028 & 0.31 & $0.12-0.83$ & 0.019 \\
\hline Smoking history & Nolyes & 0.35 & $0.14-0.91$ & 0.031 & 0.75 & $0.21-2.67$ & 0.654 \\
\hline EGFR status & Exon $19 \mathrm{del} /$ others & 0.31 & $0.12-0.79$ & 0.014 & 0.28 & $0.10-0.77$ & 0.013 \\
\hline IL-6 change types* & Reduced/nonreduced & 0.36 & $0.11-1.14$ & 0.083 & 0.52 & $0.14-1.95$ & 0.335 \\
\hline
\end{tabular}

Note: *Patients were dichotomized based on the change type during gefitinib treatment.

Abbreviations: $\mathrm{Cl}$, confidence interval; EGFR, epidermal growth factor receptor; HR, hazard ratio; IL, interleukin.

Table S2 Univariate and multivariate survival analyses involving posttreatment plasma IL-6

\begin{tabular}{|c|c|c|c|c|c|c|c|}
\hline \multirow[t]{3}{*}{ Variables } & \multirow[t]{3}{*}{ Category } & \multicolumn{6}{|c|}{ Progression-free survival analyses } \\
\hline & & \multicolumn{3}{|c|}{ Univariate analysis } & \multicolumn{3}{|c|}{ Multivariate analysis } \\
\hline & & HR & $95 \% \mathrm{Cl}$ & $P$-value & HR & $95 \% \mathrm{Cl}$ & $P$-value \\
\hline Gender & Female/male & 0.25 & $0.09-0.69$ & 0.008 & 0.23 & $0.06-0.82$ & 0.024 \\
\hline Age & $<57 / \geq 57$ years & 0.39 & $0.17-0.90$ & 0.028 & 0.30 & $0.11-0.81$ & 0.017 \\
\hline Smoking history & No/yes & 0.35 & $0.14-0.91$ & 0.031 & 1.69 & $0.45-6.45$ & 0.439 \\
\hline EGFR status & Exon $19 \mathrm{del} /$ others & 0.31 & $0.12-0.79$ & 0.014 & 0.28 & $0.10-0.76$ & 0.013 \\
\hline IL-6 level* & Lower/higher & 0.57 & $0.25-1.33$ & 0.200 & 0.47 & $0.16-1.33$ & 0.153 \\
\hline
\end{tabular}

Note: *Patients were dichotomized based on the median value $(1.7 \mathrm{pg} / \mathrm{mL})$ of posttreatment plasma IL-6.

Abbreviations: $\mathrm{Cl}$, confidence interval; EGFR, epidermal growth factor receptor; HR, hazard ratio; IL, interleukin. 

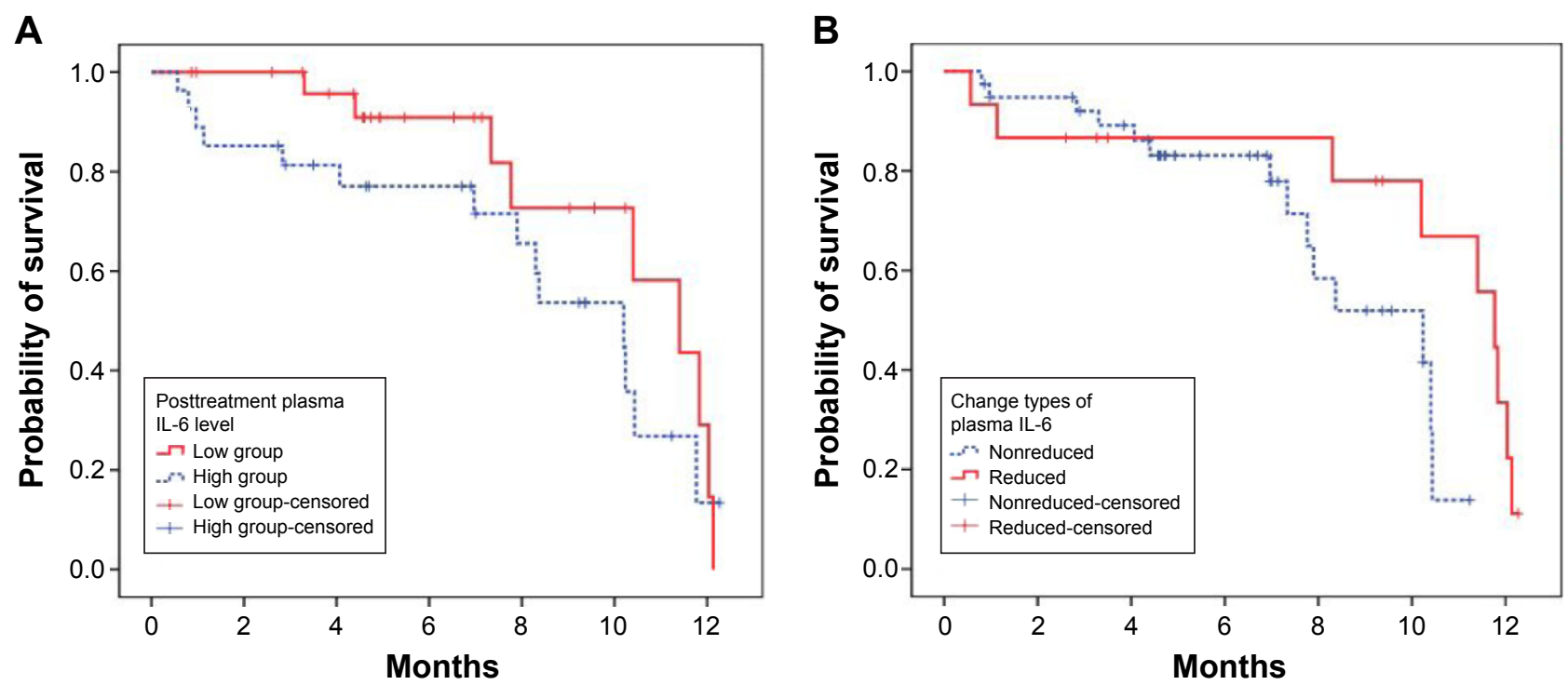

Figure S2 The prognostic value of IL-6 for progression-free survival.

Note: Higher level $(\geq 1.7 \mathrm{pg} / \mathrm{mL})$ versus lower level $(<1.7 \mathrm{pg} / \mathrm{mL})$ of IL-6 $(\mathbf{A})$ and IL-6 reduced versus nonreduced after gefitinib treatment $(\mathbf{B})$.

Abbreviation: IL, interleukin.
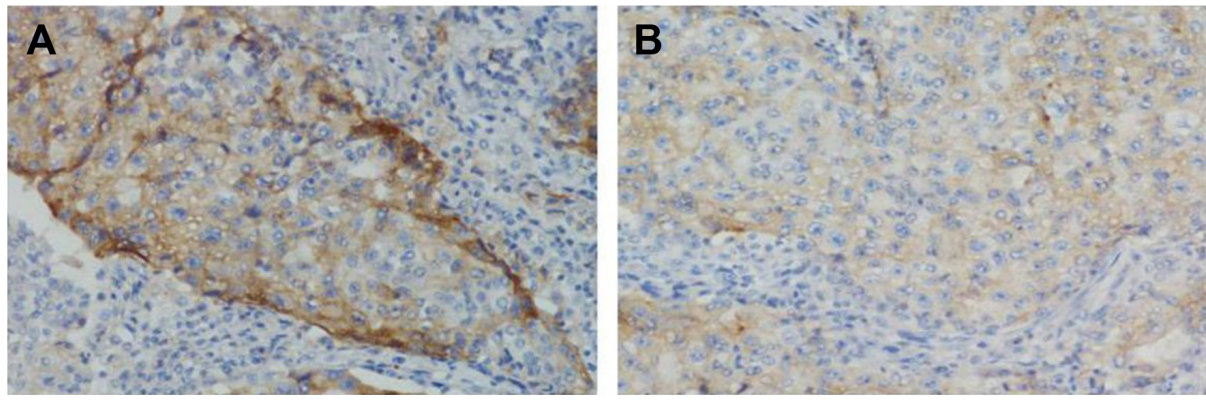

Figure S3 The expression of PD-LI by immunohistochemical staining with a membranous pattern.

Note: Baseline staining (A) and 4 weeks after gefitinib treatment (B).

Abbreviation: PD-LI, programmed cell death ligand-I.

Table S3 The levels of peripheral lymphocytes at baseline or after 4 weeks of gefitinib treatment

\begin{tabular}{|c|c|c|c|}
\hline $\begin{array}{l}\text { Lymphocyte } \\
\text { subgroup }\end{array}$ & $\begin{array}{l}\text { Percent at } \\
\text { baseline (\%) }\end{array}$ & $\begin{array}{l}\text { Percent after } \\
4 \text { weeks (\%) }\end{array}$ & $P$-value \\
\hline $\mathrm{CD}^{+} \mathrm{T}$ cells & $36.5 \pm 9.1$ & $34.4 \pm 9.2$ & 0.003 \\
\hline $\mathrm{CD}^{+} \mathrm{T}$ cells & $22.3 \pm 7.3$ & $22.6 \pm 7.4$ & 0.48 \\
\hline CD4/CD8 ratio & $1.8 \pm 0.7$ & $1.7 \pm 0.7$ & 0.08 \\
\hline NK cells & $21.3 \pm 10.0$ & $23.7 \pm 10.9$ & 0.005 \\
\hline $\mathrm{CD}^{+}{ }^{+} \mathrm{CD} 25^{+} \mathrm{T}$ cells & $13.7 \pm 5.3$ & $14.8 \pm 7.0$ & 0.18 \\
\hline
\end{tabular}

Note: The results of percent of lymphocytes of PBMC are presented as mean \pm standard deviation.

Abbreviations: NK, natural killer; PBMC, peripheral blood mononuclear cell.

OncoTargets and Therapy

\section{Publish your work in this journal}

OncoTargets and Therapy is an international, peer-reviewed, open access journal focusing on the pathological basis of all cancers, potential targets for therapy and treatment protocols employed to improve the management of cancer patients. The journal also focuses on the impact of management programs and new therapeutic agents and protocols on

\section{Dovepress}

patient perspectives such as quality of life, adherence and satisfaction The manuscript management system is completely online and includes a very quick and fair peer-review system, which is all easy to use. Visit http://www.dovepress.com/testimonials.php to read real quotes from published authors. 